\title{
Polymeric Lids for Microcontainers for Oral Protein Delivery
}

Mazzoni, Chiara; Jacobsen, Rasmus Due; Mortensen, Jacob; Jørgensen, Jacob Rune; Vaut, Lukas; Jacobsen, Jette; Gundlach, Carsten; Müllertz, Anette; Nielsen, Line Hagner; Boisen, Anja

\section{Published in:}

Macromolecular Bioscience

Link to article, DOI:

10.1002/mabi.201900004

Publication date:

2019

Document Version

Peer reviewed version

Link back to DTU Orbit

Citation (APA):

Mazzoni, C., Jacobsen, R. D., Mortensen, J., Jørgensen, J. R., Vaut, L., Jacobsen, J., Gundlach, C., Müllertz, A., Nielsen, L. H., \& Boisen, A. (2019). Polymeric Lids for Microcontainers for Oral Protein Delivery. Macromolecular Bioscience, 19(5), [1900004]. https://doi.org/10.1002/mabi.201900004

\section{General rights}

Copyright and moral rights for the publications made accessible in the public portal are retained by the authors and/or other copyright owners and it is a condition of accessing publications that users recognise and abide by the legal requirements associated with these rights.

- Users may download and print one copy of any publication from the public portal for the purpose of private study or research.

- You may not further distribute the material or use it for any profit-making activity or commercial gain

- You may freely distribute the URL identifying the publication in the public portal 


\section{Polymeric Lids for Microcontainers for Oral Protein Delivery}

Chiara Mazzoni ${ }^{1, *}$, Rasmus Due Jacobsen ${ }^{1 \#}$, Jacob Mortensen ${ }^{1 \#}$, Jacob Rune Jørgensen ${ }^{2}$, Lukas Vaut $^{1}$, Jette Jacobsen ${ }^{2}$, Carsten Gundlach ${ }^{3}$, Anette Müllertz ${ }^{2}$, Line Hagner Nielsen ${ }^{1, *}$, Anja Boisen ${ }^{1}$

${ }^{\#}$ The authors equally contributed to the work

${ }^{1}$ Department of Micro- and Nanotechnology, Technical University of Denmark, Ørsteds Plads 345C, Kgs. Lyngby, 2800, Denmark

${ }^{2}$ Department of Pharmacy, University of Copenhagen, Universitetsparken, 2, Copenhagen, 2100,

Denmark

${ }^{3}$ Department of Physics, Technical University of Denmark Fysikvej 307, Kgs. Lyngby, 2800,

Denmark

${ }^{*}$ Corresponding authors: Department of Micro- and Nanotechnology, Technical University of Denmark, Ørsteds Plads 345C, Kgs. Lyngby, 2800, Denmark.

Email: Lihan@nanotech.dtu.dk Phone: +45 45255751 (L.H. Nielsen)

Email: chimaz@nanotech.dtu.dk Phone:+45 45255732 (C. Mazzoni)

Keywords: polymeric coating, mucoadhesion, permeation enhancer, oral drug delivery, protein, microdevices 


\begin{abstract}
Oral delivery of proteins and peptides is one of the main challenges in the pharmaceutical drug development. Microdevices have the possibility to protect the therapeutics until release is desired, avoiding losses by degradation. One type of such microdevices is polymeric microcontainers. In this study, lysozyme was chosen as model protein and loaded into microcontainers along with the permeation enhancer sodium decanoate (C10). The loaded microcontainers were sealed and functionalized by applying polymeric lids onto the cavity of the devices. The first lid was poly lactic-co-glycolic acid (PLGA) and on top of this either polyethylene glycol (PEG) or chitosan was applied (PLGA+PEG or PLGA+chitosan, respectively). The functionalization was evaluated in vitro for morphology, drug release and mucoadhesive properties. These were coupled with in vitro and ex vivo studies using Caco-2 cells, Caco-2/HT29-MTX-E12 co-cultures and porcine intestinal tissue.
\end{abstract}

PLGA+chitosan showed a slower release in buffer and slower transport of lysozyme across cell cultures compared to PLGA+PEG or only PLGA. Microcontainers coated with chitosan or PEG demonstrated a three times stronger adhesion during ex vivo mucoadhesion studies compared to samples without coatings. Altogether, functionalized microcontainers with mucoadhesive properties and tunable release for oral protein delivery have been developed and characterized. 


\section{Introduction}

Macromolecules such as proteins are used for treatment of diseases such as cancer ${ }^{[1]}$, rheumatoid $\operatorname{arthritis}^{[2]}$ and psoriasis ${ }^{[3]}$, and are mainly administered by injections. To increase patient compliance and reduce costs for the health care system, it would be beneficial to deliver proteins and peptides via the oral route. ${ }^{[4]}$ Nevertheless there are certain disadvantages, which are related to their instability in the gastro-intestinal (GI) tract, poor absorption due to their large size, their hydrophilicity and proteolysis by enzymes found in the GI tract. ${ }^{[5]}$

For succeeding with oral delivery of proteins, permeation enhancers are necessary to increase intestinal absorption. ${ }^{[5]}$ These compounds allow the passage of proteins across the epithelium by opening the tight junctions. Fatty acids, medium chain glycerides and chitosan are among the most common examples of permeation enhancers. ${ }^{[5]}$ Sodium decanoate $(\mathrm{C} 10)$ is a saturated fatty acid and is well-known to open tight junctions and can thereby lead to a higher transport of large hydrophilic compounds through the epithelial cell layer. ${ }^{[6,7]}$

Moreover, in the last decades, hydrogels, nanoparticles and microparticles have been developed and studied to deliver proteins and peptides orally. ${ }^{[8]}$ The major obstacles for these delivery systems are the instability, polydispersity and the control on the amount of drug that can be loaded. ${ }^{[8]}$ Microfabricated devices such as planar reservoirs with a diameter of $200 \mu \mathrm{m}$ and a height of 4-8 $\mu \mathrm{m}$ have been used as carriers for insulin showing an enhanced drug permeability in vitro and in vivo. ${ }^{[9]}$ The authors highlighted that in these microdevices only about 50 ng of insulin was loaded and, thus, an excessive number of microdevices are needed to be administered per day. Microcontainers are polymeric cylindrical microdevices with a diameter and height of approximately $300 \mu \mathrm{m}$ having a volume 100 times larger than the reservoirs, consequently, they have a higher chance of providing a therapeutic dose. The main advantages of the devices compared to traditional particulates are that the devices 1) protect the protein from the GI environment and 2) provide unidirectional release that 
allows the delivery of the protein to a specific part of the intestine e.g. the small intestine where absorption often occurs. ${ }^{[10]}$ Previously, it has been shown that small molecules, such as furosemide and ketoprofen in microcontainers, coated with $\mathrm{pH}$-sensitive coatings improve the relative oral bioavailability of 220 and $180 \%$, respectively compared to controls. ${ }^{[11,12]}$

For obtaining optimal performance of the devices, the microcontainers can be functionalized by applying polymeric layers as lids on top of the microcontainers. Poly lactic-co-glycolic acid (PLGA) has been widely used as degradable carrier for oral protein delivery. ${ }^{[13,14]}$ Insulin-loaded PLGA nanoparticles and microcapsules have been reported to reduce the glucose level substantially in diabetic rats. ${ }^{[15,16]}$ In an aqueous environment, PLGA degrades by hydrolysis to lactic and glycolic acid, both easily metabolized by the body. ${ }^{[13]}$ During this process, the local environment in e.g. the intestine becomes more acidic and, therefore, the solubility of a proteins like insulin can be enhanced. ${ }^{[17,18]}$ Mucoadhesive polymers can prolong the residence time of the carriers at the site of absorption. ${ }^{[19]}$ Chitosan, a mucoadhesive polymer, has been used as coatings or carriers for microand nanoparticles for delivery of proteins improving their oral bioavailability. ${ }^{[8]}$ At the same time as having mucoadhesive properties it is also a permeation enhancer. ${ }^{[20,21]}$ Polyethylene glycol (PEG) has also been widely utilized for oral delivery of proteins. ${ }^{[8]}$ Tobío et al. showed that microparticles of the degradable carrier polylactic acid (PLA) coated with PEG provided a five times higher oral bioavailability of tetanus toxoid than particles without coating. This was explained to be due to the mucus penetrating effect of PEG. ${ }^{[22]}$

In this study, microcontainers loaded with the model protein lysozyme and C10 were functionalized with polymeric lids to be tested in vitro and ex vivo for oral protein delivery. The microcontainers were functionalized by applying two layers as lids on top of the microcontainers, the first layer was PLGA and on top of this, either PEG or chitosan was applied (PLGA+PEG or PLGA+chitosan, respectively). The functionalized microcontainers were evaluated in vitro for morphology and drug 
release and, ex vivo for mucoadhesive properties. Furthermore, it was assessed if the functionalization of the microcontainers improved lysozyme transport in vitro and ex vivo using cell models and porcine intestinal tissue.

\section{Results and discussion}

\subsection{Functionalization of microcontainers}

The physical powder mixture of lysozyme and C10 (7:3) was loaded into the microcontainers (Figure 1a) with an average load per chip of $1.98 \pm 0.36 \mathrm{mg}$ corresponding to each microcontainer loaded with $3.1 \pm 0.6 \mu$ g powder mixture (Figure 1b). The first functionalization layer was prepared with PLGA to help the dissolution of the protein. ${ }^{[13]}$ It was observed that the polymer was homogenously distributed over the microcontainers, although, the shape of the lysozyme powder was still visible (Figure 1c). On top of the PLGA layer, either a layer of PEG (Figure 1d-e) or chitosan (Figure 1f-g) was deposited as penetrating/mucoadhesive polymers. In both cases, after applying the second layer, the powder loaded into the microcontainers was completely covered and both coatings were smooth (Figure 1e and g). Moreover, considering the X-Ray $\mu \mathrm{CT}$ images, it was possible to conclude that the two layers were uniformly distributed on the chips (Figure 1d and $\mathbf{f}$ ). The thicknesses of the different coatings with only one or two layers were measured using an optical profilometer (Table 1). Chitosan resulted to be the thinnest among all the layers which is due to the fact that the chitosan solution was prepared in a lower concentration compared to PLGA and PEG solutions and the spraying parameters were different. Moreover, all the measurements showed that the layers were homogeneously distributed within the same chip and among chips $(\mathrm{n}=$ 3, each measured in three points). 


\subsection{In vitro drug release from functionalized microcontainers}

The release of lysozyme from the coated microcontainers showed that for the three different coatings (PLGA, PLGA+chitosan and PLGA+PEG), the loaded protein was released within $14 \mathrm{~h}$ (Figure 2). In the insert of Figure 2, it is noticeable that the different coatings resulted in different release profiles. The lysozyme released from the microcontainers coated with PLGA+chitosan reached $70.6 \pm 6.6 \%$ within the first $2 \mathrm{~h}$ of the experiment. Microcontainers coated with only PLGA or PLGA+PEG showed a faster release $(92.7 \pm 4.6 \%$ and $93.3 \pm 3.7 \%$, respectively) in the first 2 h. Slower release from the PLGA coating can be achieved by utilizing PLGA standards with either higher molecular weight or larger ratios of lactide. ${ }^{[31]}$ In accordance, it has been shown that coatings of PLGA+chitosan can induce a sustained release due to the low solubility of chitosan at $\mathrm{pH} 7.4^{[32,33]}$ as this was also observed here for the microcontainers coated with these two polymers. In the literature, chitosan nanoparticles loaded with lysozyme showed a sustained release due to the swelling of the particles resulting in $20 \%$ of lysozyme released over 3 weeks. ${ }^{[34]}$ Chitosan as a lid will also have a tendency to swell due to the hydrogel properties of chitosan ${ }^{[35,36]}$ resulting in a slower diffusion process for lysozyme from the microcontainers causing a sustained release.

\subsection{Ex vivo mucoadhesion studies}

Mucoadhesion studies applying intestinal porcine tissue were utilized to test if the top layer of the lid (chitosan or PEG) was adding a mucoadhesive feature to the microcontainers. Control of humidity and temperature (80 \% relative humidity and $\left.37^{\circ} \mathrm{C}\right)$ during the whole experiment allowed for mimicking the physiological environment, avoiding that the tissue dried out during the experiments (Figure S1). ${ }^{[5]}$ The number of microcontainers adhering to the tissue after 5 min of flow was considered a measure for mucoadhesiveness of the microcontainers. Figure $\mathbf{3}$ shows that in the presence of PEG or chitosan, the percentage of microcontainers adhering to the tissue was 
higher (40.9 $\pm 5.5 \%$ and $35.7 \pm 14.1 \%$, respectively) than for the microcontainers without coating or with only PLGA $(13.0 \pm 2.3 \%$ and $19.3 \pm 8.8$, respectively). This means that the number of microcontainers coated with either PEG or chitosan adhering to the tissue is 2-fold higher compared to the ones only coated with PLGA. If, instead, the number of samples coated with PLGA+PEG or PLGA+chitosan is compared with microcontainers without coating, they resulted to be three times more mucoadhesive. In particular, the number of microcontainers with PLGA+PEG layers that adhered was found to be statistically different from the ones having only one layer of PLGA (p = 0.0097). The high variability in these experiments can be caused by the use of different pieces of intestinal tissue having different mucus thicknesses that can lead to different adherence values. ${ }^{[37]}$

\subsection{In vitro transport studies}

Transport studies were realized using Caco-2 cell monolayers or Caco-2/HT29-MTX-E12 coculture monolayers as in vitro models for absorption through the small intestine. The Caco-2 cells are intensively used as an in vitro model for the intestinal epithelial barrier to determine the permeability of drug compounds through the intestinal barrier. ${ }^{[38]}$ However, Caco-2 cells are lacking the goblet cells responsible for mucus production. ${ }^{[39]}$ In vivo, the mucus layer acts as a physical and chemical defense from the luminal content and is important for the prediction of intestinal permeability of compounds, having a great importance for the absorption of proteins. ${ }^{[40]}$ For this reason, the co-cultivation of Caco-2 and HT-29 cell monolayers are widely used since it provides a model constituting the two cell types that are most represented in the intestinal epithelium in humans, enterocytes and goblet cells. ${ }^{[41]}$

The percentage of transported lysozyme versus time across the Caco-2 monolayer or the co-culture was calculated. The profiles for the lysozyme confined in the microcontainers are similar to those of the bulk powder (Figure 4). Although, the microcontainers coated with PLGA+chitosan showed a 
lag-time due to a slower transport of lysozyme. The average flux (Figure S2) was calculated for each analyzed sample using Equation 1. The microcontainers coated with PLGA and PLGA+PEG displayed flux values of $5.3 \pm 1.4 * 10^{-6} \mathrm{nmol} / \mathrm{min} / \mathrm{cm}^{2}$ and $4.9 \pm 1.6 * 10^{-6} \mathrm{nmol} / \mathrm{min} / \mathrm{cm}^{2}$, respectively, hence, there was no significant difference between their flux values (p-value 0.0803). The slower onset in lysozyme transport for the chitosan samples (Figure 4) is likely due to slower release of lysozyme. This delayed transport can be due to a delayed release of lysozyme from the microcontainers as showed in the in vitro studies (Figure 2) in which after 2 h, only $70.6 \pm 6.6 \%$ was released. Moreover, it has previously been shown that the presence of chitosan in insulin nanoparticles resulted in a slower transport of insulin across Caco-2 monolayers compared to other formulations. ${ }^{[20]}$ Determination of transepithelial electrical resistance (TEER) before and after the experiments allowed investigation of the integrity of the cell layer. The initial TEER values for all the cells were between 215-255 $\Omega * \mathrm{~cm}^{2}$ (Figure S2a). At the end of the experiments, all TEER values, except for the blank, dropped to $90-120 \Omega * \mathrm{~cm}^{2}$ (Figure S2a). This complies with previous findings in the literature, observing that TEER is reduced across Caco-2 cells following treatment with chitosan ${ }^{[42]}$ and especially $\mathrm{C} 10^{[6,7]}$ as they are both permeation enhancers. Thereby, they are interrupting tight junctions between the cells, but it is a reversible process and studies have shown that the cells recover within $24 \mathrm{~h}^{[7,42]}$

The transport of lysozyme from the microcontainers release across the Caco-2/HT29-MTX-E12 coculture was slower than the bulk powder (Figure 4b). The calculated lysozyme flux for PLGA, PLGA+PEG and PLGA+chitosan coated microcontainer were $6.2 \pm 3.5 * 10^{-6}, 2.7 \pm 1.2 * 10^{-6}$ and $3.9 \pm 0.8 * 10^{-6} \mathrm{nmol} / \mathrm{min} / \mathrm{cm}^{2}$, respectively. The flux seemed to be highly depended on the rate of release from the microcontainers since PLGA+PEG and PLGA+chitosan showed a slower transport and a lower flux. This is indicated by the relatively high flux seen for the controls compared to the flux for coated microcontainers. The initial TEER values for Caco-2/HT29-MTX-E12 cell 
monolayers were at consistent levels in the range of $345-390 \Omega * \mathrm{~cm}^{2}$ (Figure S2b). TEER values dropped for all the samples at the end of the experiments $(2 \mathrm{~h})$, however not significantly for the blank (Figure S2b). The microcontainers with the chitosan layer showed its permeation enhancing properties having a lower TEER values compared to the other coated with PEG or only PLGA.

The lowering of the TEER values for PLGA+chitosan, in both type of cells, highly indicated chitosan interaction with the cell monolayer. The lysozyme flux for the PLGA+chitosan coated microcontainers was observed to be higher across the co-culture than Caco-2 cell monolayers $(\mathrm{p}=$ 0.0101). A reason for this could be the presence of mucus and PEG-chitosan's known ability to form hydrogels. ${ }^{[43,44]}$ This could allow slower diffusion of lysozyme through the coating. ${ }^{[5]}$ Generally, it seems that the onset of the transport depends on the dissolution of lysozyme from the microcontainers.

\subsection{Ex vivo transport studies}

Ex vivo transport studies are more representative of the intestinal barrier compared to the use of cell-culture models in which only few types of cells are present compared to the real tissue. ${ }^{[40]}$ Hence, to mimic the in vivo situation as much as possible, intestinal transport studies were also carried out using porcine intestinal tissue in a side-by-side diffusion chamber.

The absorption of lysozyme was insufficient for chitosan coatings and a flux could not be calculated. This is believed to be caused by the slow release from the chitosan coating, due to the formation of the chitosan hydrogel, retaining the lysozyme. ${ }^{[44]}$ This hydrogel behavior was very clear from a visual inspection after the experiment. Moreover, it has been shown that chitosan can bind and cross-link mucin and mucus gel and therefore, delay the diffusion of molecules through the chitosan/mucus gel. ${ }^{[45]}$ From the tissue integrity evaluation at the end of the experiment (Figure S3), it is noticeable that the $\mathrm{P}_{\mathrm{app}}$ for PLGA+chitosan is higher than the other samples. This might be 
due to the fact that chitosan and C10 worked as permeation enhancers even though no lysozyme flux was observed. It is important to notice that, even after $180 \mathrm{~min}$, the concentration of lysozyme in the donor chamber was 10 times lower than for the other samples (data not shown) meaning that the lysozyme was not fully dissolved yet. However, a flux of lysozyme was detected for the other samples and was $3.9 \pm 0.4 * 10^{-6} \mathrm{nmol} / \mathrm{min} / \mathrm{cm}^{2}$ for the control (not coated), $7.6 \pm 1.6 * 10^{-6}$ for PLGA and $5.3 \pm 0.3 * 10^{-6} \mathrm{nmol} / \mathrm{min} / \mathrm{cm}^{2}$ for PLGA+PEG (Figure 5). The microcontainers coated only with PLGA showed a higher flux probably due to the lower thickness of the coating compared to samples coated with the second layer of PEG.

\section{Conclusion}

This work demonstrated that microcontainers can be functionalized by applying two different layers on top of the cavity of the microdevices. Since PLGA is known as a degradable polymer to enhance the solubility of the protein, it has been chosen as first layer. PEG or chitosan represented the second layer as mucoadhesive or mucus penetrating polymers. Both PLGA+PEG and PLGA+chitosan resulted in homogenous coatings on top of the lysozyme loaded microcontainers. The microcontainers with chitosan coating showed a slower lysozyme release compared to PLGA+PEG or PLGA coatings. With regard to the ex vivo mucoadhesive studies, it was found that the microcontainers coated by chitosan or PEG showed a 3-fold increase in porcine tissue adhesion, compared to the microcontainers without coatings. The in vitro studies with Caco-2 and Caco2/HT29-MTX-E12 co-culture monolayers and the ex vivo studies confirmed the results from the release study: chitosan-coated microcontainers result in a slower transport of lysozyme.

In this study, we obtained mucoadhesive microcontainers with tunable release for oral protein delivery. The functionalization will be further tested in vivo for better understanding. 


\section{Experimental section}

\section{$\underline{\text { 4.1. Materials }}$}

Silicon (Si) wafers (4-in, b100N n-type) were provided by Okmetic (Vantaa, Finland), and SU-8 2075 and SU-8 developer were purchased from Microresist Technology GmbH (Berlin, Germany). Lyophilized lysozyme (from chicken egg white, $14.8 \mathrm{kDa})(\geq 40.000$ units/mg, $90 \%$ purity), phosphate buffered saline (PBS) tablets, chitosan (low MW 50-190 kDa, 75-85 \% deacetylation), poly lactic-co-glycolic acid (PLGA) (low MW 7-17 kDa, 50:50 PLA:PGA) were obtained from Sigma-Aldrich (St. Louis, MO, USA). 10x Hank’s balanced salt solution (HBSS) and sodium bicarbonate were obtained from Thermo Fisher Scientific (Hampton, NH, USA), whereas polyethylene glycol (PEG) (low MW 12 kDa) was purchased from Fisher Scientific (Hampton, NH, USA). n-Capric acid sodium salt (C10) was obtained from abcr GmbH (Karlsruhe, Germany). 4-(2hydroxyethyl)-1-piperazineethanesulfonic acid (HEPES), bovine serum albumin (BSA) and Dulbecco’s Modified Eagle’s Medium - high glucose, acetonitrile, trifluoroacetic acid (TFA), dichloromethane (DCM) and acetic acid (100.5\%) were all acquired from Sigma-Aldrich (St. Louis, MO, USA). D-[1-14C]-mannitol was obtained from Perkin Elmer (Waltham, MA, USA). Milli-Q deionized water was provided by a MilliQ Integral Water Purification System for Ultrapure Water, produced by Merck Millipore (Burlington, MA, USA).

\subsection{Fabrication of microcontainers}

Microcontainers were fabricated in the epoxy-based photoresist SU-8 using a procedure similar to the one described earlier. ${ }^{[23,24]}$ A fluorocarbon coating was deposited on top of the supporting silicon wafer by plasma polymerization to ease the detachment of microcontainers from the chip. ${ }^{\text {[11] }}$ The dimensions of the microcontainers were measured using an Alpha-Step IQ Stylus Profilometer (KLA-Tencor Corporation, Milpitas, USA) and optical microscopy. The fabricated microcontainers 
had an inner diameter of $232 \pm 1 \mu \mathrm{m}$ and a height of $255 \pm 6 \mu \mathrm{m}$ (mean $\pm S D, n=4)$ (Figure 1a). After fabrication, the wafers were cut into squared chips of 1.2 x $1.2 \mathrm{~cm}$ containing 625 microcontainers using an Automatic Dicing Saw from DISCO (Kirchheim b. München, Germany).

\subsection{Loading of lysozyme into microcontainers}

To facilitate the loading of lysozyme, the received powder was lightly ground in a mortar to obtain a fine powder. Lysozyme was carefully mixed with C10 (7:3 w/w ratio). A shadow mask was attached on top of the chip to cover the areas in between the microcontainers and to avoid excess powder in these spaces. ${ }^{[25]}$ The fine powder of lysozyme was evenly distributed on top of the mask and pressed into the cavity of the microcontainers with a brush. The shadow mask was removed together with the excess of powder resulting in filled microcontainers. The chips with microcontainers were weighed before and after filling to assess the amount of loaded powder.

\subsection{Functionalization of microcontainers}

After loading, the coatings were applied onto the cavity of the microcontainers to functionalize them. The coating was performed by spray coating with an ExactaCoat Ultrasonic Spray System (Sonotek, USA) with an accumist nozzle operating at $120 \mathrm{kHz}$. The polymer solutions used were: PLGA (0.7 \%) in DCM (w/v), PEG (0.7 \%) in DCM (w/v) or chitosan (0.5 \%) in acetic acid (0.1 M, w/v). The PLGA lid was sprayed followed by either PEG or chitosan on top (PLGA+PEG or PLGA+chitosan, respectively). Microcontainers only with a PLGA lid were also investigated. During the procedure, the flow rate was kept at $0.1 \mathrm{~mL} / \mathrm{min}$, together with a generator power of 1.3 W. Each chip was coated with two alternating spray paths having an offset of $1 \mathrm{~mm}$, resulting in a total of 60, 120 or 70 passages for PLGA, chitosan or PEG, respectively. The shaping air, the speed of the nozzle and the distance between the nozzle and the sample are described in Table 2. For the 
aqueous acidic solution used for the chitosan coating, the plate underneath the chip was set to a temperature of $40^{\circ} \mathrm{C}$ during the spraying process.

\subsection{Morphology characterization}

The samples PLGA+PEG and PLGA+chitosan were subjected to X-ray micro computed tomography (X-ray $\mu \mathrm{CT}$ ) investigations using a commercial Zeiss Xradia versa 410 system (Pleasanton, USA) to assess the coating morphology. The samples were mounted on a pin such that undisturbed $360^{\circ}$ access to the sample was obtained. The pre-voltage was kept at $60 \mathrm{kV}$ and the power at $10 \mathrm{~W}$. The $4 \mathrm{X}$ optical objective was chosen resulting in an efficient pixel size of $2.83 \mu \mathrm{m}$, 3201 projection angles were measured over $360^{\circ}$. The exposure time for each projection was $4 \mathrm{~s}$ and the total scanning time $5 \mathrm{~h}$ and $2 \mathrm{~min}$. All collected data were reconstructed by the commercial software connected to the system which relies on a Feldkamp, Davis and Kress algorithm, ${ }^{[26]}$ based on filtered back-projection algorithm. Three areas from each sample were analyzed to obtain a more representative image of the whole chip.

The loading and coating processes were investigated using a Tabletop Microscope TM3030 (Hitachi High-Technologies Europe GmbH, Krefeld, Germany). The samples were observed at a $30^{\circ}$ tilt using $15 \mathrm{kV}$ and secondary electrons (SE) detector.

Flat silicon chips with a layer of SU-8 on top were sprayed with each polymer solution for thickness measurements as described in the "Functionalization of microcontainers" section. However, these were only coated on one half, by placing a flat half chip on top. The thickness measurements were performed with a KLA-Tencor Alpha-Step IQ stylus profilometer (Milpitas, CA, USA) with a scan speed of $50 \mu \mathrm{m} / \mathrm{s}$ and force of $15.3 \mathrm{mg}$. Each chip was measured in three different places (middle and sides). 


\subsection{In vitro drug release from functionalized microcontainers}

The release of lysozyme from the spray coated microcontainers was studied using a $\mu$-DISS profiler (pION INC, Woburn, MA) connected to a temperature controlled water bath (Struers Kebo Lab, Rødovre, Denmark). The release of lysozyme from the coated microcontainers (with PLGA, PLGA+PEG and PLGA+chitosan) was performed attaching each chip to a cylindrical magnetic stirrer and covering it with PBS (10 mL of $10 \mathrm{mM})$ at $\mathrm{pH} 7.4$ at $100 \mathrm{rpm}$. The temperature was 37 ${ }^{\circ} \mathrm{C}$ and the absorbance was measured at $280 \mathrm{~nm}$. The path length of the UV probes was $20 \mathrm{~mm}$ and each channel was calibrated with concentrations ranging from 0.03 to $0.3 \mathrm{mg} / \mathrm{mL}$. The percentage of release was calculated from the known amount of powder loaded per chip. These experiments were replicated from 5 to 6 times for microcontainers coated with PLGA, PLGA+PEG or PLGA+chitosan.

\subsection{Ex vivo mucoadhesion studies}

An ex vivo setup similar to the one described in literature ${ }^{[27,28]}$ was used to perform the experiments for investigating the mucoadhesion of the coated microcontainers. Porcine intestine was obtained from healthy experimental control pigs (50-55 kg, 15-16 weeks of age, LYD pigs). Immediately after euthanasia of the pigs, the intestines were excised using a scalpel and placed in ice. Within $2 \mathrm{~h}$, the tissues were frozen and on the day of the experiment, a $5 \mathrm{~cm}$ piece was cut longitudinally and placed on the supporting slide with the apical side facing upwards. The mucosal side visually looked intact including the mucus layer as the intestine was frozen without preparation.

A pump (Watson Marlow, Falmouth, United Kingdom) was used to apply a peristaltic flow of PBS buffer $(10 \mathrm{mM})$ at $\mathrm{pH}$ 7.4. The used setup was temperature and humidity controlled with a temperature of $37^{\circ} \mathrm{C}$ and relative humidity of $80 \%$ (Figure S1). Initially, the loosely adhering mucus was washed away for $1 \mathrm{~min}$ with a flow of $10 \mathrm{~mL} / \mathrm{min}$. After coating the chips, the 
microcontainers were gently manually scraped off the silicon chip. A defined amount of microcontainers $(177 \pm 35)$ were applied onto a spatula to ease the placement of them on the middle of the porcine intestinal tissue (in the flow path). Subsequently, a flow of $10 \mathrm{~mL} / \mathrm{min}$ was applied for $5 \mathrm{~min}$, after which the intestine was left to dry for $24 \mathrm{~h}$. The remaining microcontainers were counted using a Leica S9E stereo microscope (Leica, Wetzlar, Germany) to determine the percentage of microcontainers that adhered to the intestinal tissue at the end of the experiment. This experiment was repeated with three different pieces of tissue for every type of sample (empty microcontainers, microcontainers with PLGA, PLGA+PEG and PLGA+chitosan coatings).

\subsection{In vitro transport studies}

The Caco-2 (HTB-37) cell line and Caco-2/HT29-MTX-E12 co-cultured cell monolayers were acquired by American Type Culture Collection (Manassas, VA, USA) and Inserm (Paris, France), respectively. The Caco-2 and the co-culture cells had been used in the number of passages of 37-43 and 65-71, respectively. All the cells were cultured as described by M. Natoli et al., ${ }^{[29]}$ and transport experiments were performed on polarized cells after 21 days on permeable supports. In vitro transport studies were carried out in 6 well plates, and before the experiment, each plate was left at room temperature for 20 min. Subsequently, TEER was measured for each well and all wells were afterwards washed twice with HBSS buffer (1X HBSS, HEPES (10 mM), BSA (0.05 \% w/v), sodium bicarbonate $(0.04 \% \mathrm{w} / \mathrm{v}))$ with a $\mathrm{pH}$ of 7.4 . Following this, HBSS buffer was added on the apical side $(1.5 \mathrm{~mL})$ and to the basolateral side $(2.6 \mathrm{~mL})$. Each chip was then gently placed upside down directly onto the cell layers. The experiment was initiated when the plates were put on a table shaker set at $75 \mathrm{rpm}$ and $37{ }^{\circ} \mathrm{C}$. Samples were taken $(100 \mu \mathrm{L})$, and the volume was replaced with pre-heated HBSS, from the basolateral side at specific time points of 15, 30, 45, 60, 90 and 120 min. A single sample was also taken from the apical side after $120 \mathrm{~min}$. After the $2 \mathrm{~h}$ runtime, the 
chips were gently removed. The cells were washed twice with HBSS buffer and TEER values were measured again. All samples were frozen at $-20{ }^{\circ} \mathrm{C}$ and analyzed with the RP-HPLC methods described in "Reversed-phase High-performance Liquid Chromatography (RP-HPLC) method". The lysozyme flux $(F)\left[\mathrm{mol} / \mathrm{cm}^{2} / \mathrm{s}\right]$ was calculated according to Equation 1.

$$
F=\frac{d Q}{d t} \cdot \frac{1}{A}
$$

where $\mathrm{dQ} / \mathrm{dt}[\mathrm{mol} / \mathrm{s}]$ is the rate of lysozyme permeation and $\mathrm{A}$ is the area of the inserts $\left(4.67 \mathrm{~cm}^{2}\right)$. All the experiments were done in triplicates.

\subsection{Ex vivo transport studies}

Ex vivo transport studies were performed using a modular EM-CSY-8 Ussing chamber system (Physiologic Instruments, San Diego, CA, USA) including a temperature controlled metal rack for mounting Ussing chambers in series. Ussing chambers containing a vertical port were assembled and mounted onto the rack. A slider was inserted between each pair of chambers and a volume of HBSS buffer $(2 \mathrm{~mL})$ at $\mathrm{pH} 7.4$ was added to each chamber. The metal rack was pre-heated to equilibrate the system until the buffer in the chambers reached $35-37{ }^{\circ} \mathrm{C}$. Moreover, humidified airflow was applied to each chamber. A piece of porcine intestine was carefully stripped to remove the serosa and the longitudinal and circular muscle layers of the intestine. ${ }^{[30]}$ After equilibration, the buffer was removed, the chambers were dried and the slider was taken out. A small cut of stripped intestine was carefully stretched and mounted into the slider by impaling the edges of the intestine on a circle of small metal pins around the aperture. Loaded and coated microcontainers were scraped off and gently applied onto the apical side of the intestine before the slider was re-inserted into the Ussing chambers. Pre-heated HBSS buffer $(2 \mathrm{~mL}) \mathrm{pH} 7.4$ was then added to each chamber. Samples were taken from the receptor side $(100 \mu \mathrm{L})$ at $30 \mathrm{~min}$ time intervals from 0 min to 180 
min. A single sample was also taken from the donor side $(100 \mu \mathrm{L})$ at $0 \mathrm{~min}$ and $180 \mathrm{~min}$. All samples were analyzed with RP-HPLC described in "Reversed-phase High-performance Liquid Chromatography (RP-HPLC) method" and the flux was calculated as described in the "In vitro transport studies" section. Following each ex vivo transport experiment, the integrity of the porcine intestine was evaluated by the flux of radioactive D-[1- $\left.{ }^{14} \mathrm{C}\right]$-mannitol. A HBSS buffer with D-[1$\left.{ }^{14} \mathrm{C}\right]$-mannitol $(0.1 \mathrm{mM})$ was prepared, and the solution $(2 \mathrm{~mL})$ was added to the apical side and regular HBSS buffer was added to the basolateral side $(2 \mathrm{~mL}$ ). Three samples (of each $100 \mu \mathrm{L}$ ) were taken at 0 and $60 \mathrm{~min}$ from both the apical and basolateral side. Three samples were also taken from the original D-[1- $\left.{ }^{14} \mathrm{C}\right]$-mannitol $(0.1 \mathrm{mM})$ HBSS buffer solution. Ultima Gold ${ }^{\mathrm{TM}}$ Scintillation fluid (2 mL) was added to each sample, vortexed, and analyzed by a scintillation analyzer (PerkinElmer, Tri-Carb® 2910 TR). The apparent permeability coefficient $\left(P_{a p p}\right)[\mathrm{cm} / \mathrm{s}]$ has been calculated according to Equation 2.

$$
P_{a p p}=\frac{d Q}{d t} \cdot \frac{1}{A \cdot C_{0}}=\frac{F}{C_{0}}
$$

where $d Q / d t$ [mol/s] is the rate of lysozyme permeation, $A$ is the area of the inserts $\left(0.78 \mathrm{~cm}^{2}\right), C_{0}$ $\left[\mathrm{mol} / \mathrm{cm}^{3}\right]$ is the initial donor concentration of $\mathrm{D}-\left[1-{ }^{14} \mathrm{C}\right]-$ mannitol and $F$ is described in (1).

All the experiments were repeated four times per sample type except for the microcontainers coated with PLGA and chitosan which were tested in triplicate.

\subsection{Reversed-phase High-performance Liquid Chromatography (RP-HPLC) method}

Samples obtained from the in vitro and ex vivo transport studies were analyzed by RP-HPLC on a Prominence Ultra-Fast Liquid Chromatography (UFLC) instrument system (Shimadzu, Japan). The column used was a Kinetex $5 \mu \mathrm{m}$ XB-C18 (100 Å, 100 x 4.6 mm) (Phenomenex, USA). The mobile phases were acetonitrile with TFA $(0.1 \%$, v/v) (solvent A) and MilliQ deionized water with TFA $(0.1 \%, \mathrm{v} / \mathrm{v})$ (Solvent B) at $30{ }^{\circ} \mathrm{C}$ and solvent $\mathrm{A}$ had a steady gradient from $29 \%$ to $71 \%$ over 15 
min at a flow rate of $1.4 \mathrm{~mL} / \mathrm{min}$, and the absorbance was measured at $280 \mathrm{~nm}$. RP-HPLC has been used also to verify the integrity of the protein after grinding and leaving it at room temperature for one week (data not shown).

\subsection{Statistical analysis}

Data is expressed as mean \pm standard error of the mean (SEM) for ex vivo mucoadhesion studies and ex vivo and in vivo transport studies. Mean \pm standard deviation (SD) has been used in all the other data analysis. P-values are calculated using the unpaired t-test in GraphPad Prism (GraphPad Software, CA, USA) and were considered statistically significant when below $5 \%(\mathrm{p}<0.05)$.

\section{Acknowledgments}

The authors would like to acknowledge the Center for Intelligent Drug Delivery and Sensing Using Microcontainers and Nanomechanics (IDUN) whose research is funded by the Danish National Research Foundation (DNRF122) and Villum Fonden (Grant No. 9301). The 3D Imaging Centre at The Technical University of Denmark is gratefully acknowledged. Associate Professor Stephan Sylvest Keller is acknowledged for the help with fabrication of the microcontainers and fruitful discussions regarding the spray coating technique. Laboratory technicians Lene Grønne Pedersen and Mette Frandsen from University of Copenhagen are both acknowledged for their help regarding the cell cultivation.

\section{Conflict of interest}

The authors declare no conflict of interest 


\section{References}

[1] D. Peer, J. M. Karp, S. Hong, O. C. Farokhzad, R. Margalit, R. Langer, Nat. Nanotechnol. 2007, 2, 751.

[2] P. C. Taylor, Curr. Opin. Pharmacol. 2003, 3, 323.

[3] M. Patel, A. Day, R. B. Warren, A. Menter, Dermatol. Ther. (Heidelb). 2012, 2, 16.

[4] M. Morishita, N. A. Peppas, Drug Discov. Today 2006, 11, 905.

[5] A. M. Wagner, M. P. Gran, N. A. Peppas, Acta Pharm. Sin. B 2018, 8, 147.

[6] T. Lindmark, N. Schipper, L. Lazorová, A. G. De Boer, P. Artursson, J. Drug Target. 1998, 5, 215.

[7] A. C. Chao, J. V. Nguyen, M. Broughall, A. Griffin, J. A. Fix, P. E. Daddona, Int. J. Pharm. 1999, 191, 15.

[8] D. J. McClements, Adv. Colloid Interface Sci. 2018, 253, 1.

[9] C. B. Fox, C. L. Nemeth, R. W. Chevalier, J. Cantlon, D. B. Bogdanoff, J. C. Hsiao, T. A. Desai, Bioeng. Transl. Med. 2017, 2, 9.

[10] B. F. Choonara, Y. E. Choonara, P. Kumar, D. Bijukumar, L. C. du Toit, V. Pillay, Biotechnol. Adv. 2014, 32, 1269.

[11] L. H. Nielsen, A. Melero, S. S. Keller, J. Jacobsen, T. Garrigues, T. Rades, A. Müllertz, A. Boisen, Int. J. Pharm. 2016, 504, 98.

[12] C. Mazzoni, F. Tentor, S. A. Strindberg, L. H. Nielsen, S. S. Keller, T. S. Alstrøm, C. Gundlach, A. Müllertz, P. Marizza, A. Boisen, J. Control. Release 2017, 268, 343.

[13] F. Danhier, E. Ansorena, J. M. Silva, R. Coco, A. Le Breton, V. Préat, J. Control. Release 2012, 161, 505.

[14] C. Snider, S.-Y. Lee, Y. Yeo, G. J. Grégori, J. P. Robinson, K. Park, Pharm. Res. 2008, 25, 5. 
[15] S. Sun, N. Liang, H. Piao, H. Yamamoto, Y. Kawashima, F. Cui, J. Microencapsul. 2010, 27, 471.

[16] B. S. Kim, J. M. Oh, H. Hyun, K. S. Kim, S. H. Lee, Y. H. Kim, K. Park, H. B. Lee, M. S. Kim, Mol. Pharm. 2009, 6, 353.

[17] B. S. Zolnik, D. J. Burgess, J. Control. Release 2007, 122, 338.

[18] O. Siddiqui, Y. Sun, J.-C. Liu, Y. W. Chien, J. Pharm. Sci. 1987, 76, 341.

[19] S. K. Lai, Y.-Y. Wang, J. Hanes, Adv. Drug Deliv. Rev. 2009, 61, 158.

[20] A. M. M. Sadeghi, F. A. Dorkoosh, M. R. Avadi, M. Weinhold, A. Bayat, F. Delie, R. Gurny, B. Larijani, M. Rafiee-Tehrani, H. E. Junginger, Eur. J. Pharm. Biopharm. 2008, 70, 270.

[21] I. A. Sogias, A. C. Williams, V. V. Khutoryanskiy, Biomacromolecules 2008, 9, 1837.

[22] M. Tobío, A. Sánchez, A. Vila, I. Soriano, C. Evora, J. . Vila-Jato, M. . Alonso, Colloids Surfaces B Biointerfaces 2000, 18, 315.

[23] P. Marizza, S. S. Keller, A. Boisen, Microelectron. Eng. 2013, 111, 391.

[24] L. H. Nielsen, S. S. Keller, K. C. Gordon, A. Boisen, T. Rades, A. Müllertz, Eur. J. Pharm. Biopharm. 2012, 81, 418.

[25] Z. Abid, C. Gundlach, O. Durucan, C. von Halling Laier, L. H. Nielsen, A. Boisen, S. S. Keller, Microelectron. Eng. 2017, 171, 20.

[26] L. A. Feldkamp, L. C. Davis, J. W. Kress, J. Opt. Soc. Am. A 1984, 1, 612.

[27] K. D. Madsen, C. Sander, S. Baldursdottir, A. M. L. Pedersen, J. Jacobsen, Int. J. Pharm. 2013, 448, 373.

[28] K. V. R. Rao, P. Buri, Int. J. Pharm. 1989, 52, 265.

[29] M. Natoli, B. D. Leoni, I. D’Agnano, A. Felsani, Toxicol. Vitr. 2012, 26, 1243.

[30] L. L. Clarke, Am. J. Physiol. Gastrointest. Liver Physiol. 2009, 296, G1151. 
[31] G. Mittal, D. K. Sahana, V. Bhardwaj, M. N. V. Ravi Kumar, J. Control. Release 2007, 119, 77.

[32] N. Ahmad, M. A. Alam, R. Ahmad, S. Umar, F. Jalees Ahmad, J. Microencapsul. 2018, 1.

[33] K. Tahara, T. Sakai, H. Yamamoto, H. Takeuchi, Y. Kawashima, Int. J. Pharm. 2008, 354, 210.

[34] A. M. Piras, G. Maisetta, S. Sandreschi, S. Esin, M. Gazzarri, G. Batoni, F. Chiellini, Int. J. Biol. Macromol. 2014, 67, 124.

[35] F. Ahmadi, Z. Oveisi, S. M. Samani, Z. Amoozgar, Res. Pharm. Sci. 2015, 10, 1.

[36] M. R. Rekha, C. P. Sharma, in Pept. Protein Deliv. (Ed: C. VanDerWalle), Elsevier, 2011, pp. 165-194.

[37] M. P. D. Gremião, F. C. Carvalho, M. L. Bruschi, R. C. Evangelista, M. Palmira, D. Gremião, Brazilian J. Pharm. Sci. 2010, 46.

[38] H. Sun, E. C. Chow, S. Liu, Y. Du, K. S. Pang, Expert Opin. Drug Metab. Toxicol. 2008, 4, 395.

[39] T. Lea, in Impact Food Bioact. Heal., Springer International Publishing, Cham, 2015, pp. 103-111.

[40] C. R. Kleiveland, in Impact Food Bioact. Heal., Springer International Publishing, Cham, 2015, pp. 135-140.

[41] A. Wikman-Larhed, P. Artursson, Eur. J. Pharm. Sci. 1995, 3, 171.

[42] T.-H. Yeh, L.-W. Hsu, M. T. Tseng, P.-L. Lee, K. Sonjae, Y.-C. Ho, H.-W. Sung, Biomaterials 2011, 32, 6164.

[43] S. Lu, K. S. Anseth, Macromolecules 2000, 33, 2509.

[44] S. Yan, T. Wang, X. Li, Y. Jian, K. Zhang, G. Li, J. Yin, RSC Adv. 2017, 7, 17005.

[45] S. Kootala, L. Filho, V. Srivastava, V. Linderberg, A. Moussa, L. David, S. Trombotto, T. 
Crouzier, Biomacromolecules 2018, 19, 872. 


\section{Tables and Figures:}

Table 1: Thicknesses of the different coatings either as individual coatings or combined with PLGA. The data represent the mean of three samples measured in three different points of a chip (central and sides) \pm standard deviation (SD).

\begin{tabular}{cc}
\hline Coating & Thickness $[\boldsymbol{\mu m}]$ \\
\hline PLGA & $17.1 \pm 2.4$ \\
PEG & $17.0 \pm 5.6$ \\
PLGA+PEG & $32.5 \pm 8.0$ \\
Chitosan & $6.9 \pm 1.1$ \\
PLGA+chitosan & $22.5 \pm 3.8$ \\
\hline
\end{tabular}

Table 2: Parameters used for spray coating PLGA, chitosan and PEG onto the cavity of microcontainers.

\begin{tabular}{cccc}
\hline & PLGA & Chitosan & PEG \\
\hline Shaping air [kPa] & 0.03 & 0.01 & 0.03 \\
Nozzle speed [mm/s] & 10 & 25 & 10 \\
Distance between the & & & \\
nozzle and the sample & 50 & 25 & 50 \\
[mm] & & & \\
\hline
\end{tabular}




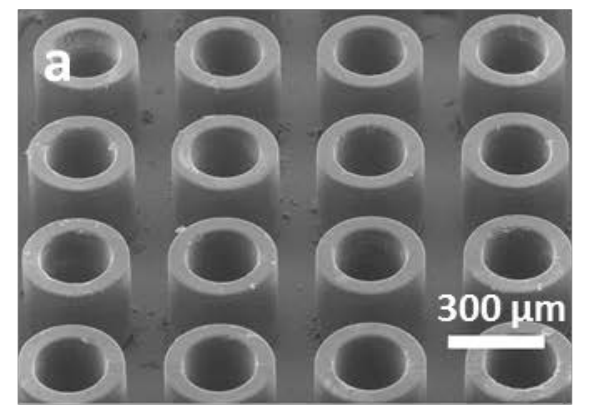

d

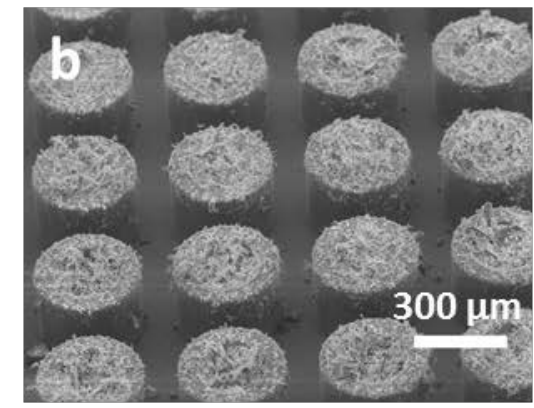

20.
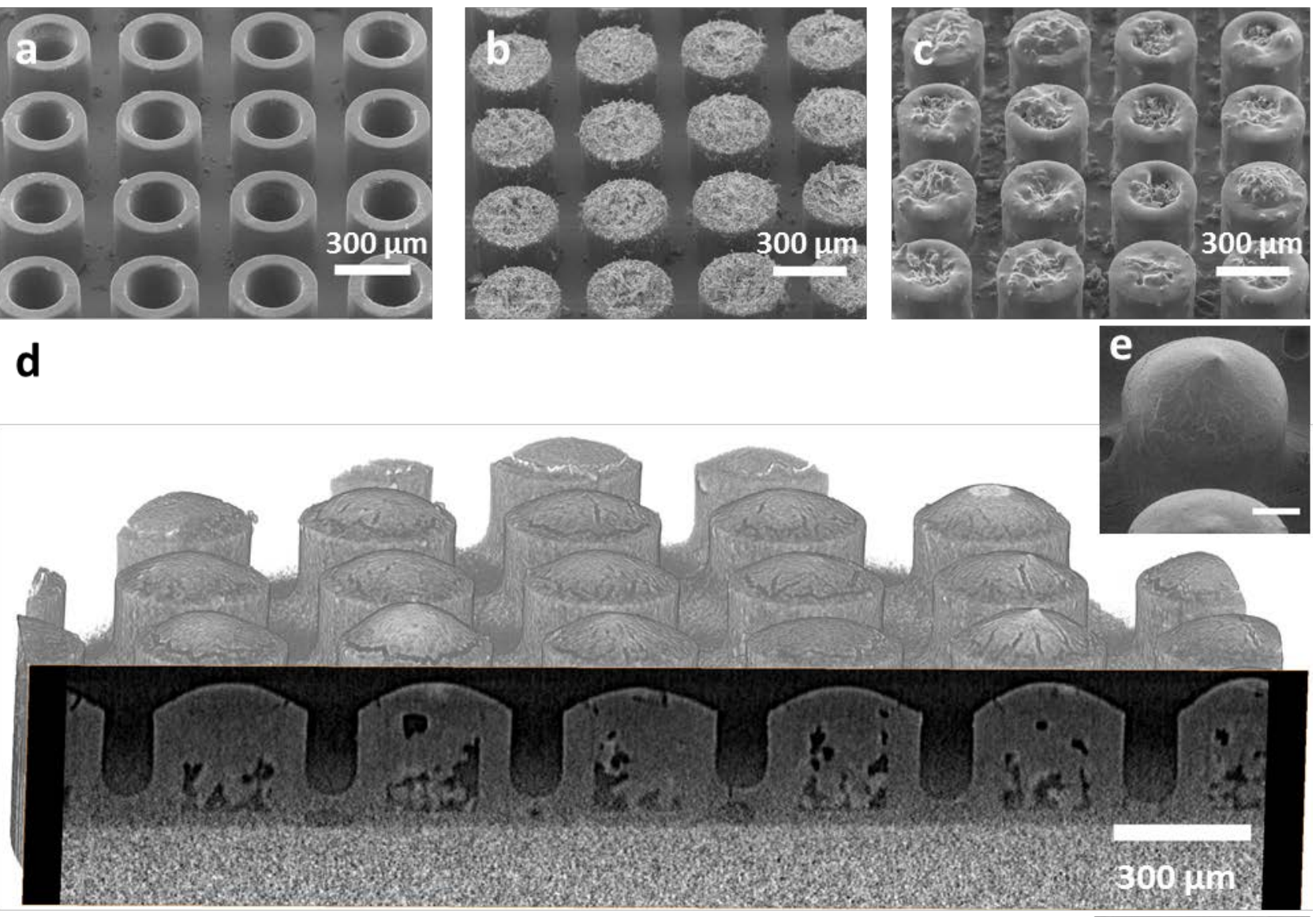

f
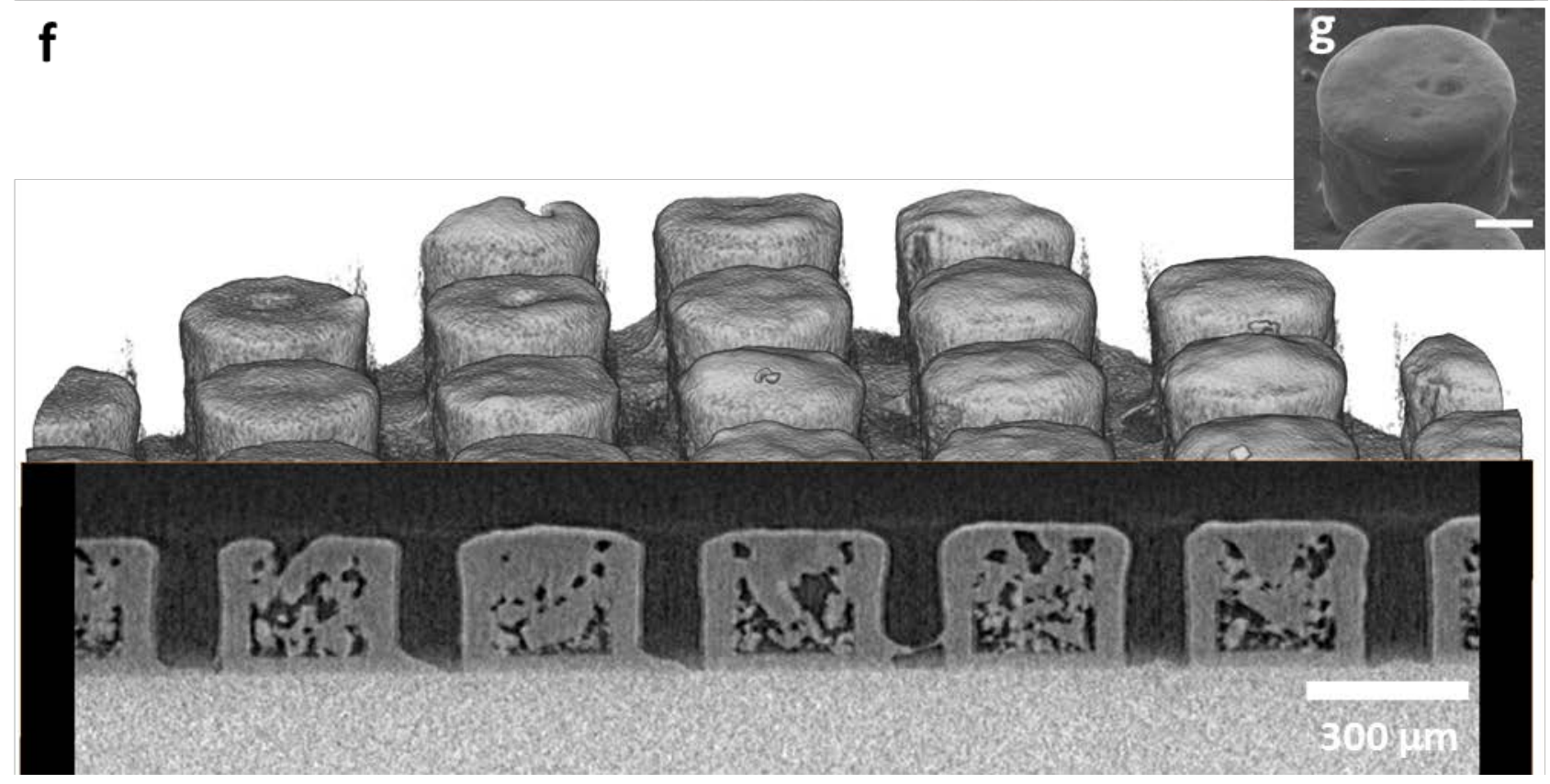

Figure 1: SEM images of a) empty microcontainers, b) microcontainers loaded with lysozyme and C10 (7:3 w/w) and c) loaded microcontainers coated with PLGA. 
$\mathrm{X}$-Ray $\mu \mathrm{CT}$ cross sections and reconstruction of loaded microcontainers coated with PLGA and on top of it either d) chitosan or f) PEG. The same samples are illustrated with SEM pictures in e) and g). The scale bars, where not indicated, correspond to $100 \mu \mathrm{m}$.

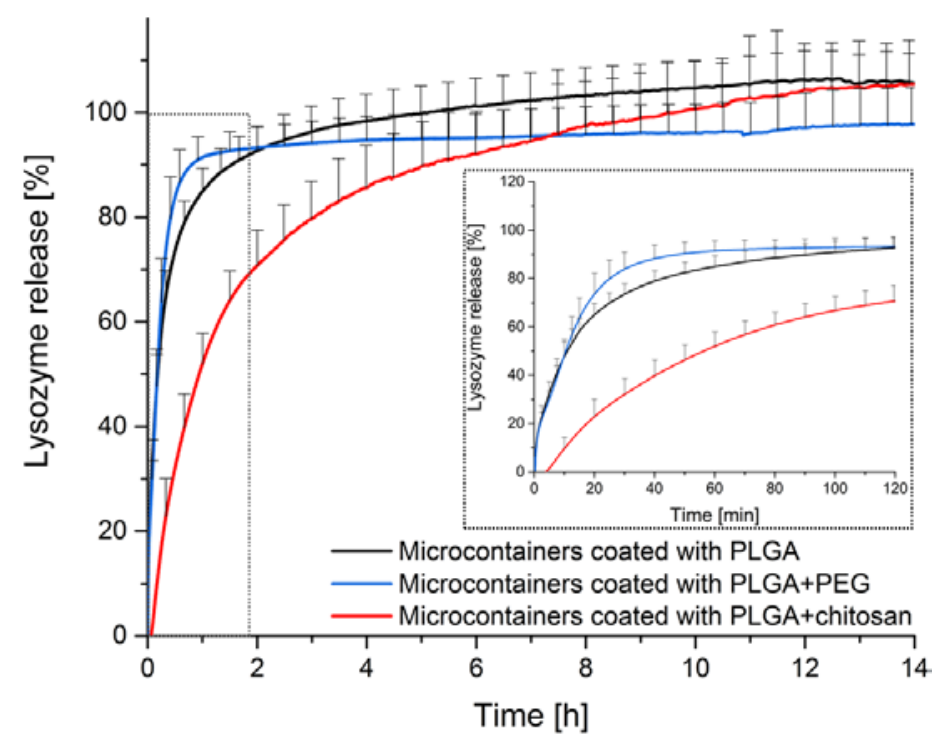

Figure 2: In vitro release of lysozyme from microcontainers with only PLGA, with PLGA+PEG and with PLGA+chitosan coatings. The release was performed on a $\mu$-DISS profiler in $10 \mathrm{mM}$ PBS at $\mathrm{pH} 7.4$ and $37^{\circ} \mathrm{C}$. The data represent mean $+\mathrm{SD}, \mathrm{n}=5-6$.

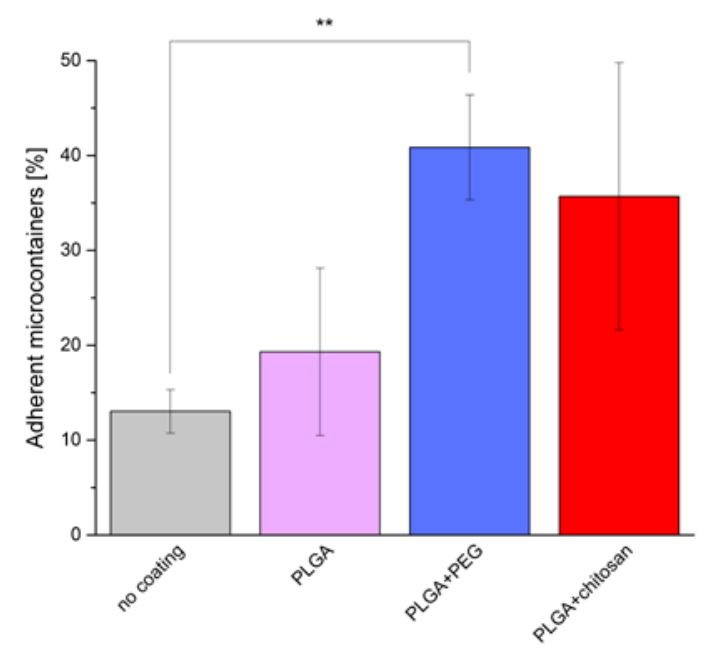


Figure 3: Percentage of microcontainers adhering to intestinal porcine tissue after flow for 5 min with $10 \mathrm{mM}$ PBS at $\mathrm{pH}$ 7.4. The microcontainers were loaded with lysozyme and were either without coating, coated with only PLGA, PLGA+PEG or PLGA+chitosan. The graph represents mean $\pm \operatorname{SEM}(\mathrm{n}=3)$.
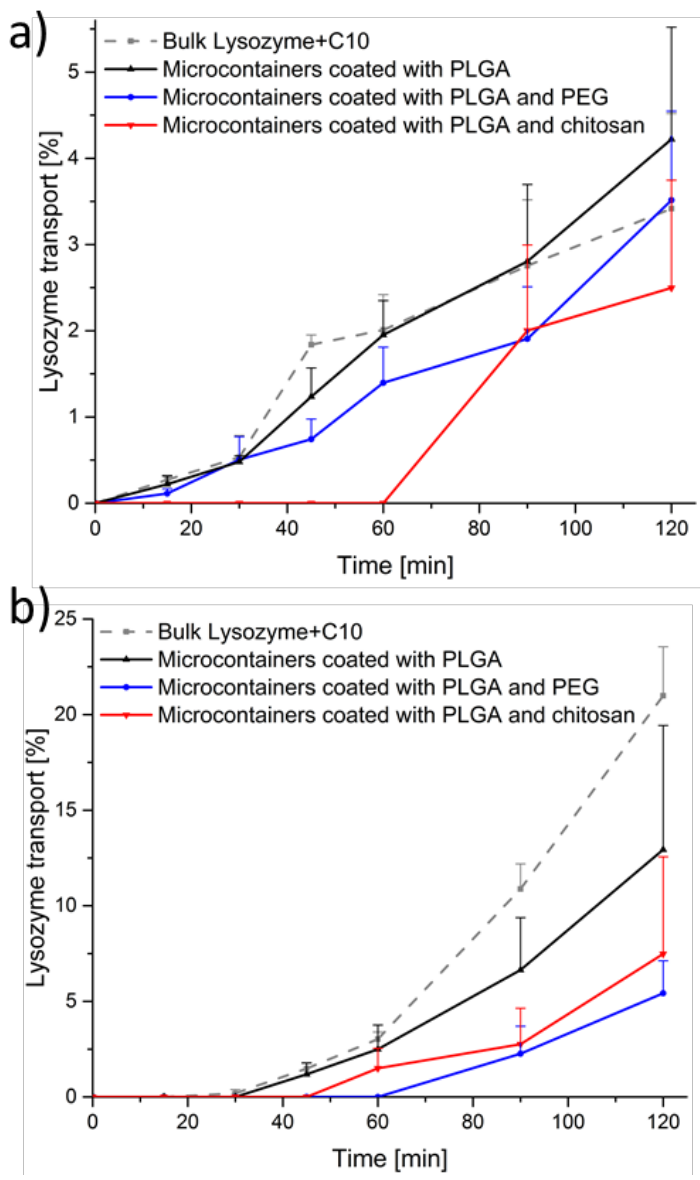

Figure 4: The transport of lysozyme across a) Caco-2 and b) Caco-2/HT29-MTX-E12 cell monolayers is shown for the bulk lysozyme powder and C10 and microcontainers coated with PLGA, PLGA+PEG or PLGA+chitosan. The graphs represent mean + SEM in triplicates. 


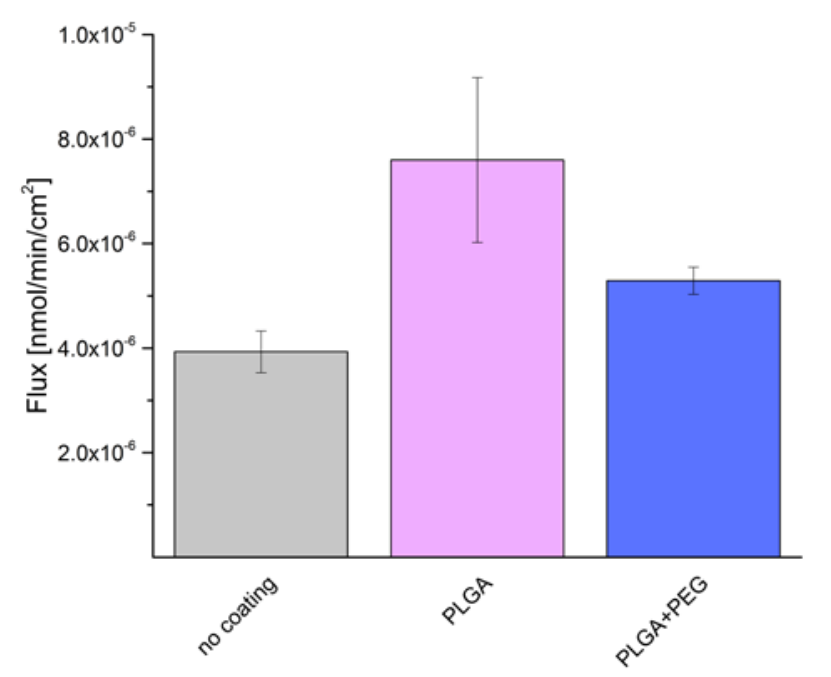

Figure 5: Flux of lysozyme across ex vivo porcine intestinal tissue. Microcontainers without coating, with PLGA and with PLGA+PEG were tested. The data are represented as mean \pm SEM with $n=4$ (microcontainers with PLGA+chitosan, $n=3$ ).

\section{Supporting Information}

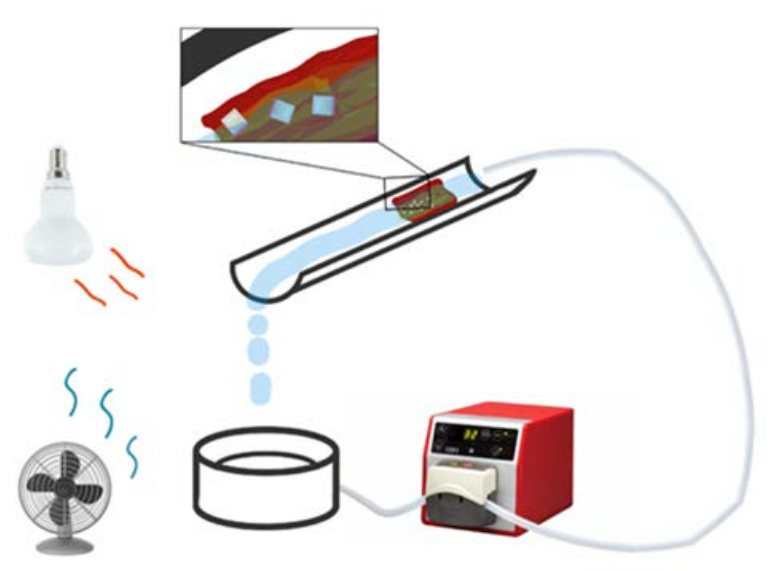

Figure S1: Schematic representation of the ex vivo mucoadhesion setup. Pig intestine is placed on a slide and flushed with a peristaltic pump. The temperature and humidity are controlled. 
a)

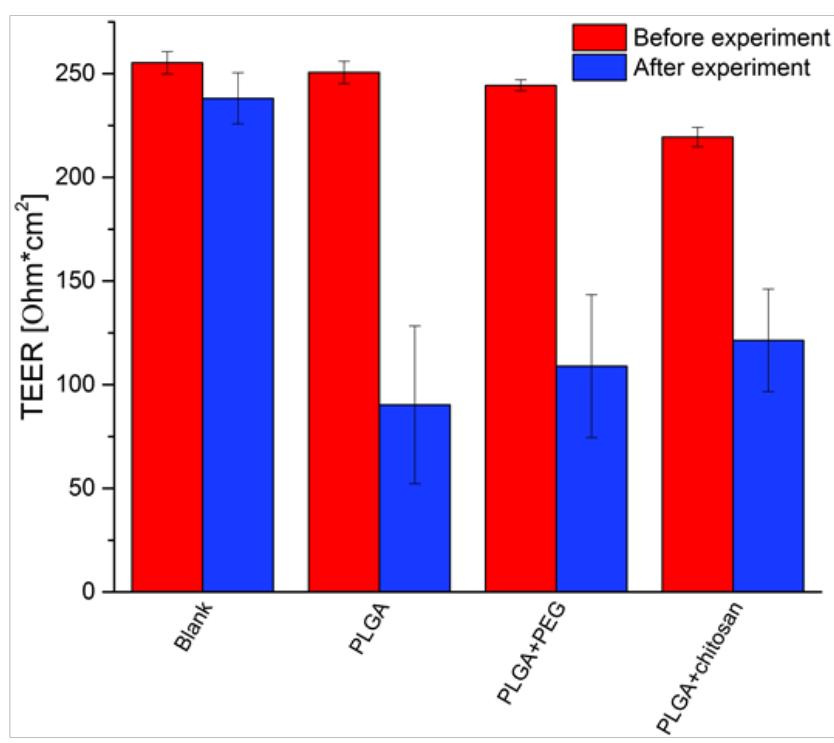

b)

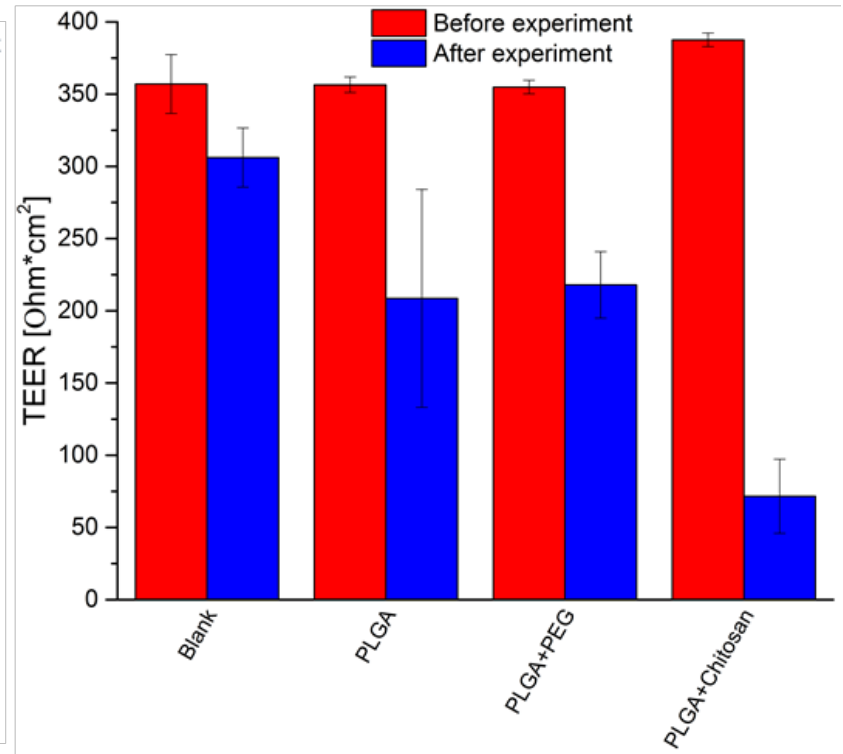

Figure S2: TEER values measured before and after the experiments for the blank, microcontainers coated with PLGA, PLGA+PEG and PLGA+chitosan. These were done with a) Caco-2 cell monolayer and b) Caco-2/HT29-MTX-E12 cell monolayers (mean \pm SD, $n=3$ ).

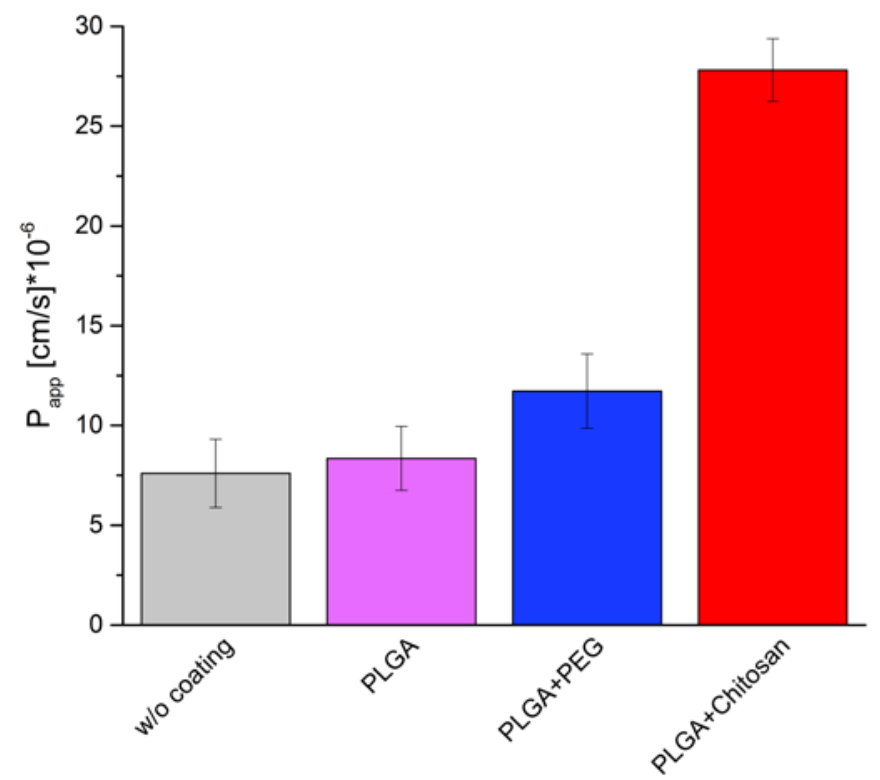

Figure S3: $\mathrm{P}$ app of D-[1- $\left.{ }^{14} \mathrm{C}\right]-$ mannitol calculated after the ex vivo transport studies comparing the microcontainers without coating with the ones with only PLGA, PLGA+PEG and PLGA+chitosan. All the values are represented as mean $\pm S D, n=2-4$. 


\section{Table of Content}

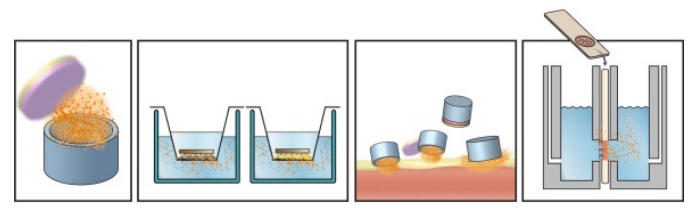

Oral delivery of proteins is one of the main challenges in the pharmaceutical field. Polymeric coatings have been applied onto microcontainers (cylindrical microdevices) to functionalize them for oral protein delivery. The coatings have been characterized in vitro and ex vivo evaluating their mucoadhesive properties and the ability to promote protein transport across intestinal barriers. 